\title{
The impact of cultural beliefs and practices on parents' experiences of bereavement following stillbirth: a qualitative study in Uganda and Kenya
}

Elizabeth Ayebare ${ }^{1 *}$, Tina Lavender ${ }^{2}$, Jonan Mweteise ${ }^{3}$, Allen Nabisere ${ }^{3}$, Anne Nendela ${ }^{4}$, Raheli Mukhwana ${ }^{4}$, Rebecca Wood ${ }^{5}$, Sabina Wakasiaka ${ }^{6}$, Grace Omoni ${ }^{6}$, Birungi Susan Kagoda ${ }^{7}$ and Tracey A. Mills ${ }^{2}$

\begin{abstract}
Background: Stillbirth is an extremely traumatic and distressing experience for parents, with profound and longlasting negative impacts. Cultural beliefs and practices surrounding death vary considerably across different contexts and groups, and are a key influence on individual experiences, impacting grief, adjustment, and support needs. Few studies have explored cultural influences surrounding stillbirth in an African context. This study explored the influence of cultural beliefs and practices on the experiences of bereaved parents and health workers after stillbirth in urban and rural settings in Kenya and Uganda.
\end{abstract}

Methods: A qualitative descriptive study design was employed. Face to face interviews were conducted with parents $(N=134)$ who experienced a stillbirth ( $\leq 1$ year) and health workers $(N=61)$ at five facilities in Uganda and Kenya. Interviews were conducted in English or the participants' local language, audio-recorded and transcribed verbatim. Analysis was conducted using descriptive thematic analysis.

Results: Commonalities in cultural beliefs and practices existed across the two countries. Three main themes were identified: 1) Gathering round, describes the collective support parents received from family and friends after stillbirth. 2)'lt is against our custom' addresses cultural constraints and prohibitions impacting parents' behaviour and coping in the immediate aftermath of the baby's death. 3) 'Maybe it's God's plan or witchcraft' summarises spiritual, supernatural, and social beliefs surrounding the causes of stillbirth.

Conclusions: Kinship and social support helped parents to cope with the loss and grief. However, other practices and beliefs surrounding stillbirth were sometimes a source of stress, fear, stigma and anxiety especially to the women. Conforming to cultural practices meant that parents were prevented from: holding and seeing their baby, openly discussing the death, memory-making and attending the burial. The conflict between addressing their own needs and complying with community norms hindered parents' grief and adjustment. There is an urgent need to develop culturally sensitive community programmes geared towards demystifying stillbirths and providing an avenue for parents to grieve in their own way.

Keywords: Culture, Qualitative, Stillbirth, Beliefs, Bereavement, East Africa, Experiences

\footnotetext{
*Correspondence: lizayeby@gmail.com; elizabeth.ayebare@mak.ac.ug

${ }^{1}$ Department of Nursing, College of Health Sciences, Makerere University,

P.O.BOX. 7072, Kampala, Uganda

Full list of author information is available at the end of the article
} original author(s) and the source, provide a link to the Creative Commons licence, and indicate if changes were made. The images or other third party material in this article are included in the article's Creative Commons licence, unless indicated otherwise in a credit line to the material. If material is not included in the article's Creative Commons licence and your intended use is not permitted by statutory regulation or exceeds the permitted use, you will need to obtain permission directly from the copyright holder. To view a copy of this licence, visit http://creativecommons.org/licenses/by/4.0/. The Creative Commons Public Domain Dedication waiver (http://creativeco mmons.org/publicdomain/zero/1.0/) applies to the data made available in this article, unless otherwise stated in a credit line to the data. 


\section{Background}

A disproportionate burden of global stillbirth, defined as death of a baby before or during birth after 28 weeks' gestation [1], affects low- and middle-income countries (LMICs). In sub-Saharan Africa and South Asia, including Uganda and Kenya, rates are around tenfold higher than high-income countries [2]. The death of a baby before or during childbirth is a traumatic and distressing experience for parents and families [3] associated with wide ranging and long-lasting negative health and social impacts. These include increased risk of recurrence, adverse mental health outcomes including anxiety, depression and post-traumatic stress disorder [4]. In LMICs, women may also experience chronic physical ill-health related to traumatic birth including obstetric fistula, in addition to the death of their baby. Culture, which is often defined as patterns of learned and shared behaviours and values evolving over generations within communities [5], is a key influence on acceptable conduct within societies. Culture influences 'norms' for spirituality, social and gender roles, attitudes towards family, work, health and illness [5, 6] and individual responses to adverse life events [5] including illness or bereavement [7]. Therefore, culture will affect parents' reactions, decision making, coping mechanisms and the support available from others when a baby is stillborn [8]. Research surrounding the impact of stillbirth, mostly conducted in high-income countries (HICs), demonstrates that the death of a baby, before or during birth is a unique experience, associated with significant social stigma and isolation. This can invalidate parent's grief experiences, hence prolonging and intensifying negative emotions. Lack of awareness and understanding amongst families and communities combine to reduce social support, which is recognised as a critical predictor of effective coping and adjustment [9].

Contrary to previous opinion, recent studies suggest that parents in sub-Saharan Africa experience similar psychological impacts and reactions to the death of a baby to those in other settings [3]. Less is known about key barriers and facilitators to parents' coping and adjustment, but cultural beliefs and practices in these settings could amplify risks for negative experiences and outcomes after the death of a baby. In a study of community perceptions of stillbirth in rural Uganda, Kiguli et al., identified rapid burial with limited rituals and restrictions on public mourning for stillborn babies [10]. Mothers were often excluded from funerals and deprived of the collective support ordinarily expected after death of a loved one in African communities. The high value placed on fertility and expectation of motherhood as a predominant role for most women, may also be problematic for parents who experience perinatal death. In some communities, miscarriage (early pregnancy losses, often referring to fetal deaths before 28 weeks of gestation) [11] and stillbirth are widely believed to stem from bad omens, witchcraft or immorality and women who experience repeated losses are frequently reported to be shunned and even abandoned by their partners and families [12].

Understanding cultural and social influences on experiences in the immediate postnatal period after the death of a baby in LMICs is crucial to improving support for bereaved parents and challenging wider stigma surrounding stillbirth. There have been very few studies of parents' experiences after the death of a baby in subSaharan Africa where the greatest numbers of perinatal deaths occur, particularly in urban communities, and health-workers' perspectives are largely absent. This study aimed to describe the influence of cultural beliefs and practices on the experiences of bereaved parents and health workers after stillbirth in urban and rural settings in Kenya and Uganda.

\section{Methods \\ Design, Participants and Study Setting}

This study was conducted as part of a larger programme of work exploring experiences after stillbirth in subSaharan Africa and employed a qualitative descriptive design. Women and male partners who had experienced a stillbirth, in five urban, peri-urban and semi-rural facilities and surrounding communities in Kenya and Uganda within the previous 12 months, were recruited. In 2019, stillbirth rates for Kenya and Uganda were reported at 19.7 and 17.8 per 1000 births, respectively [13]. Most women give birth in health facilities, but postnatal care following discharge is extremely limited, partly due to focus on child health. Health workers including midwives, nurses and doctors who regularly provided care for bereaved women, in the same facilities were also recruited. In both countries, the research teams were supported by Community Engagement and Involvement (CEI) groups of parents who had previously experienced the death of a baby, and stakeholder groups including local academics, clinicians, and policy makers. CEI and stakeholder groups contributed to the research process at all stages from design to interpretation and dissemination [14].

\section{Participant selection and recruitment}

Women were identified with the help of link midwives working at the facility maternity units. In Uganda, women who had birthed at home were additionally identified via village health team (VHT) members. Brief study information was provided and written consent to be contacted by the research team was sought. Not 
sooner than four weeks after the birth, research assistants contacted women to inquire whether they were interested in receiving more information. Those who agreed to participate were invited to an interview at a mutually convenient time, not sooner than six weeks after the birth. Male partners were recruited via women participants, midwives, VHTs and CEI group members. Where both partners in a couple agreed to participate in the research, interviews were conducted separately to provide a 'safe' space for free and open discussion around experiences, some of which might have related to the other partner. Health workers were informed about the research during meetings at the included facilities and information leaflets placed in the units. Those interested in participating, contacted the research team directly for more information. The sample size was guided by achieving data adequacy, in providing rich participant accounts across parents and health workers residing in urban and rural settings in the included countries. Data saturation was not prerequisite, although the sample size selected would be projected to be sufficient and no new codes were identified at the conclusion of analysis [15].

\section{Data collection tools and procedure}

In-depth, individual, semi-structured interviews were conducted by trained research assistants at a place chosen by the participant; for women and partners this was mostly at home. Health workers were interviewed in a private room in their place of work. Topic guides were developed by the research team based on existing literature and reviewed by CEI groups in both countries, prior to use. Interviews with women and partners were conducted in the participants' preferred language including English, Luganda, Kiswahili and Runyankole/Rukiga, health workers were interviewed in English. Interviews commenced with a broad opening question inviting participants to share experiences surrounding their baby's death, for women: 'Can you tell me about your pregnancy, the birth and the death of your baby'. Health workers were asked to describe their role and experiences with women and families after stillbirth. Additional focus was on exploring support and practices after the birth, the role of family, friends and communities including participant's own beliefs and traditions and those they were aware of in their communities. A participant-led approach was adopted by allowing interrupted responses, and using prompts such as 'can you tell me more about... or what makes you say... to clarify responses and explore in-depth where required. Field notes were made during and after each interview to capture nuances, including non-verbal cues. All interviews were audio-recorded, transcribed in the original language, and translated to
English where necessary. Independent back-translation of a sample of translated interviews (around 10\%) ensured accuracy of translation. Pseudonyms were used to protect participant identity. A study- specific distress policy was available to guide researchers in supporting participants discussing sensitive topics and emotions during data collection.

\section{Data Analysis}

Due to the sensitive nature of the subject matter, transcripts were not returned to the participants for confirmability, however recapping was carried out at the end of each interview. Interview data were analysed thematically, using a four-stage approach, as described by Braun and Clarke (2006) [16]. Firstly, transcripts were read and re-read to ensure familiarisation with the data. Notes were made on the patterns and meanings in the data in relation to the research question. Formal manual coding was performed, and codes were organised into subthemes and main themes. Initial analyses were conducted by two researchers in each country and one UK based researcher and emergent themes discussed with the wider research team research to support rigour. Lastly, final themes were discussed, agreed upon and named as presented in the results with supporting quotes. The final interpretation was confirmed after presentation and discussion with the CEI and stakeholder groups in both countries.

\section{Results \\ Demographic characteristics}

A total of 195 interviews were conducted with 75 women (Kenya $n=44$, Uganda $n=31$ ); 59 male partners (Kenya $n=41$, Uganda $n=18$ ) and 61 health workers (Kenya $n=41$, Uganda $n=20$ ) from June 2017 to May 2019. Tables 1 and 2 present a summary of the demographic characteristics for the participants.

\section{Themes}

Three main themes were identified from the interviews which described influences of cultural practices and beliefs on parent's experiences after the death of a baby, the theme titles include direct quotes from participants. Collective support was illustrated in descriptions of families and friends 'gathering round' the bereaved parents; 'it is against our custom' described cultural constraints and prohibitions impacting parents' behaviour and coping in the immediate aftermath of the baby's death; spiritual, supernatural, and social beliefs surrounding the causes of stillbirth are summarised in 'maybe it's God's plan or witchcraft'. 
Table 1 Participant characteristics Parents $(N=134)$

\begin{tabular}{|c|c|c|c|c|}
\hline \multicolumn{5}{|l|}{ Women $n=75$; Male Partners $n=59$} \\
\hline \multirow[t]{2}{*}{ Characteristic/Country } & \multicolumn{2}{|c|}{ Kenya $n=85$} & \multicolumn{2}{|c|}{ Uganda $n=49$} \\
\hline & $\begin{array}{l}\text { Women } \\
n=44\end{array}$ & $\begin{array}{l}\text { Partners } \\
n=41\end{array}$ & $\begin{array}{l}\text { Women } \\
n=31\end{array}$ & $\begin{array}{l}\text { Partners } \\
n=18\end{array}$ \\
\hline Age (years; median[range]) & 28.5 [19-43] & $31[20-51]$ & $25[19-40]$ & $34[23-63]$ \\
\hline Years of education (median [range]) & $12[4-17]$ & $15[6-20]$ & $10[3-16]$ & $11[3-17]$ \\
\hline Urban dwelling n (\%) & $19(43 \%)$ & $22(54 \%)$ & $18(49 \%)$ & $8(44 \%)$ \\
\hline Married or cohabiting $\mathrm{n}(\%)$ & $39(89 \%)$ & $40(98 \%)$ & $22(72 \%)$ & $16(89 \%)$ \\
\hline \multicolumn{5}{|l|}{ Religion n (\%) } \\
\hline Christian & $44(100 \%)$ & $38(93 \%)$ & $23(74 \%)$ & 7(39\%) \\
\hline Muslim & & $3(7 \%)$ & $8(26 \%)$ & $9(50 \%)$ \\
\hline Other/None & & & & $(11 \%)$ \\
\hline No living children & $15(34 \%)$ & $15(37 \%)$ & $9(29 \%)$ & $0(0 \%)$ \\
\hline Previous baby stillborn or neonatal death & $9(20 \%)$ & $5(12 \%)$ & $1(3 \%)$ & $2(4 \%)$ \\
\hline
\end{tabular}

\section{Gathering round}

Families and friends played a central, and largely positive, role in providing emotional and practical support after the death of a baby by 'gathering round' mothers to care for them. Beyond offering condolences and comfort in the immediate period after the death, family members often took the lead in facilitating rituals, including arranging the funeral. This was often mandated by parents' exclusion from involvement in burial of their own baby. However, some mothers, were physically unable to take part because of prolonged ill health resulting from pregnancy or birth complications. Family assistance ensured conventions could be followed and was highly valued by many parents:

"My family buried my child; they obtained a birth certificate, and they were able to bury him. I still appreciate what they did because I was in no position to attend the burial." (Bibi, mother, peri urban Kenya)

Beyond the first days and weeks after the baby's death, family and close friends continued to provide social and financial support to women, including help with domestic work, childcare, and provision of money to compensate for lost income. These networks were a crucial resource, allowing women and partners to focus on coping with the death of the baby and their own emotional recovery.

"My friends took care of me, even my family members sent me some money and we managed to survive...They used to cook for me food, they could give me that warm massage, such things. They used to keep me company at home. They could come and sleep with me here to console me... It strengthens you in a way; you slowly start to forget what happened because whenever you are alone, you spend the entire time crying, but when you are with someone, she can bring up stories and you chat, and even laugh a bit". (Nansubuga, mother, semi-rural Uganda)

For male partners, the presence of extended family and female friends was additionally perceived as important in enabling them to return to employment and resume

Table 2 Participant characteristics Health workers $(N=61)$

\begin{tabular}{|c|c|c|c|c|}
\hline Characteristic & $N=61$ & & & \\
\hline \multirow[t]{2}{*}{ Gender (n; \%) } & Female & & Male & \\
\hline & $46(75 \%)$ & & $15(25 \%)$ & \\
\hline Kenya & $33(80 \%)$ & & $8(20 \%)$ & \\
\hline Uganda & $13(65 \%)$ & & $7(35 \%)$ & \\
\hline \multirow[t]{2}{*}{ Role (n; \%) } & Nurse-Midwife & Midwife & Doctor & Other \\
\hline & $37(61 \%)$ & $12(20 \%)$ & $10(16 \%)$ & $2(3 \%)$ \\
\hline \multirow[t]{2}{*}{ Highest level of education (n; \%) } & Certificate & Degree & Postgrad degree & \\
\hline & $44(72 \%)$ & $13(21 \%)$ & $4(7 \%)$ & \\
\hline Time since qualification (years; median [range]) & $15(1-30)$ & & & \\
\hline
\end{tabular}


their primary role as provider for the family, as Maganda commented:

"Her female friends and neighbours would visit her and comfort her. When we came back here, I only stayed with her for only two days and resumed duty at work. Her female friends and neighbours came and gave her company." (Maganda, father, urban Uganda)

Women living in urban settings in both Kenya and Uganda, particularly those who had migrated for employment or partners' employment tended to describe feelings of loneliness and isolation more frequently after the death of their baby.

"I expected my husband to tell his family, but he didn't, and I have one brother who lives far so I too didn't want to tell him. We even spoke this morning, but I didn't want to tell him about it." (Lilly, mother peri-urban Kenya)

Barriers to accessing family included distance, transport costs and availability of mobile phones. In these circumstances some parents sought alternative 'communities' to avoid isolation, religion was an important focus and church members/ groups were very prominent in filling gaps.

\section{It is against our customs}

Cultural norms were cited as a key influence on the behaviour and actions of both parents and health workers after the death of a baby. These perceptions affected opportunities for seeing or having contact with the baby after the birth, whether the baby was named, mourning and burial rituals.

\section{I didn't see his face}

For some parents, decisions around whether to see or have contact with the dead baby immediately after birth were influenced by fears of going against 'customs' and potential negative effects. Fears around future fertility or recurrent pregnancy loss were commonly cited as specific reasons for not having contact. Despite this, several women expressed regret for not having the opportunity to see or hold their baby, among few opportunities to create memories of their existence.

"No, I didn't. I didn't get a chance to hold her. I think I was afraid, and I was not understanding anything, I was more confused and also it is against our customs to hold dead babies." (Joy, mother, peri-urban Kenya)
Assumptions of cultural prohibition meant that some health workers did not routinely offer the option of seeing or holding the stillborn babies to women. Others were conflicted in recognising the need to provide individualised care but had limited time and opportunity to support parents in making the 'right' decision that included being sensitive to cultural prohibitions. They expressed anxiety and uncertainty about whether their current practice was helpful:

"...someone tells you; in our culture we are not supposed to look at the dead baby. You will not, when you are trying to [show them the baby] ... much as you know the importance of them looking at the baby... you try to respect their culture and let them do what they feel is right." (Lynette, health workerUganda)

\section{They don't take it as a person}

Participants in both Kenya and Uganda related community views and perceptions that babies who were stillborn did not have the same status as individuals who were born alive. Angella, whose baby was stillborn explained:

"If it's a stillbirth they don't take it as a person, they do that only for a child who has at least cried after coming out of the womb. One who has cried is a human while the stillbirth isn't regarded as a human since they haven't heard its cry." (Angella, mother, urban Uganda)

This view of stillborn babies as 'less human' was further reinforced by use of the pronoun 'it' in some narratives and linked as a reason for the lack of normal rituals marking the baby's existence. Although a few women, predominantly in urban Kenya, referred to their baby by name in the interview, naming a stillborn baby was relatively uncommon.

"The baby has to first be born and he stays for about 2 days, that's when they give him a name. One whom God takes away from earth after being born is the one who gets a name... But with the other one [referring to a stillborn baby] remember, he is born already dead, that one doesn't get a name, he doesn't." (Obei, father semi-rural Uganda)

This reluctance to assign a name was often rooted in the practice of passing down family or traditional names. Using familial names for a dead baby was considered to likely bring bad luck to the whole family, consequently naming ceremonies and baptism were not extended to stillborn babies even where parents were religious. 


\section{We do it differently}

Parents and health workers in both countries described mourning rituals and funerals as being different for stillborn babies as compared to other deaths. Babies were buried very rapidly, often on the day after the birth, with only a few mourners present and a lack of normal traditions.

"No condolence money is collected. No money is collected as condolence fee from the community members. After burial, no last funeral rites are done. No gathering and cooking is done after burial like for older people. They take it like as if you have not lost anyone." (Mugisha, father, semi -rural Uganda).

Participants associated rapid burial with secrecy and taboos surrounding stillbirth, one father in Kenya was told it was necessary to avoid the baby's body being stolen and used for witchcraft. In some communities, fathers generally assumed responsibility for planning and conducting the burial: "We buried it [baby] without informing anyone else;
me, my friend and my dad plus my brother who
stays in Mbale. We proceeded and buried at that
time without letting the neighbours know". ... where
you bury, the grave isn't supposed to be seen; you
bury and just dig around it to camouflage around.
You can even plant there something. Only family
members are supposed to know and not all [family
members] even because if you know Shafik is a witch
doctor and has a bad heart; you don't show[him]."
(Shafik, father, urban Uganda)

However, it was also commonplace for both parents to be excluded from funerals of stillborn babies. This was explained by concerns around future fertility or potential for recurrence of pregnancy loss. For example, women and partners were told that if a woman of reproductive age participates in the burial of a stillborn baby, she may never conceive again. Often, older relatives particularly women who were past childbearing age took the lead role.

"For us, when a child dies, we [mother] aren't supposed to bury them anyway...it's a family affair." (Hellen, mother, urban Kenya)

"In our culture, when a baby dies from the mother's womb, we do it differently; it's the elderly people that bury that baby, women who have reached menopause..." (Ali, father, semi-rural Uganda)

Not being able to attend the funeral caused considerable distress for some parents. Amongst concerns was uncertainty as to whether the 'necessary' rituals had been performed and potential for future harm arising from omissions. For example, in central Uganda, traditions dictated that the umbilical cord should be cut off before burial of a stillborn baby and if this were not done, it was believed that the mother would not conceive again. Conversely, other women were forced by their circumstances to take a more active role in funerals than they wished. Lack of a willing partner, family support or resources to travel home meant women attending or conducting the burial personally. Failing to adhere to prescribed behaviour led to considerable anxiety around the potential impacts.

"I felt bad about it in that up to now, it still worries me. Because as you know, culturally, people allege that I might not be able to give birth again...the fact that if the mother buries their baby, she will never conceive again in her life." (Nalweyiso, mother, semi-rural Uganda)

The place of burial, an important and sometimes contentious issue, depended on the culture and marital status of the parents. Patriarchal traditions across Kenya and Uganda dictated that children should normally be buried at their fathers' ancestral burial grounds. These were sometimes located a distance away from the parents' current residence and some lacked resources to transport the baby's body. Access to family burial grounds was sometimes denied to single or separated women who also lacked financial resources to secure an appropriate burial and place to commemorate the baby's life and existence.

“...because they asked me where I was going to bury the child but I had nowhere, I didn't have any single coin because even... So, there is a woman who had a banana plantation in the neighbourhood, and she said that a baby who hasn't cried is buried in the middle of 2 banana plants. That was the only solution available at that time and I also didn't have anything to do about it." (Nalweyiso, mother, semirural Uganda)

In urban Kenya, some facilities offered hospitalarranged funerals or access to public burial grounds for babies who were stillborn. These options were valued by parents facing financial pressures, geographical distance, and family disagreements over where to bury the baby, such as Jolly:

\footnotetext{
"Yes, but you know I buried the baby not at our home or anywhere but at the cemetery, because there was a disagreement between our families. It was like the baby had died and among the [ tribe], they have their own cultures and we have our own cultures. I am a lady who was not married by that time and I
} 
could not take the baby to our home, and I could not take the baby to the father's place, so we had to bury the baby at the [district]cemetery." (Jolly, motherperi urban Kenya)

\section{Maybe it's God's plan or witchcraft}

Although participants acknowledged medical causes for stillbirth, spiritual, social and traditional beliefs were equally prominent. Religious beliefs featured conspicuously in most participant's narratives and provided fundamental context for accounts of human experience. Stillbirth was frequently described in terms of 'God's plan', with the lack of individual control over events offering comfort to women and partners, who often spoke of their 'gratitude' to God that the mother had survived.

"We believe that whatever happens is a decision from God because we did all that we could; the scanning was done properly, we went through antenatal, took medications, she used to sleep under a mosquito net." (Issac, father semi-rural Uganda)

Religious perspectives were also often invoked by health workers when they offered support or reassurance to parents in the immediate aftermath of the baby's death.

"So [pause] I tell them that it's not you who decided to get this pregnancy, it was God's plan, he is the one who decided that you conceive and it's the same God who has taken away this baby..." (Ritah, health worker, Uganda)

However, not all participants took comfort from the notion that the baby's death was predetermined by a higher power, several described their faith being tested. In these cases, religious fatalism contributed to feelings of powerlessness and lacking control over their lives which added to hopelessness and despair:

"I thought about many things. I said, am I really the one who gives birth to children and they die? What did I do to you God?" (Evalyne, mother, urban Uganda)

Participants in both countries also related widespread beliefs in their communities that supernatural forces were responsible for poor health outcomes including stillbirth. These beliefs unpinned links between natural disasters and poor pregnancy outcomes held in some communities:

"Maybe even the earthquake which happened when I was pregnant. When one is pregnant and by any chance an earthquake occurs, you get a miscarriage and sometimes the baby dies in-utero. And when it occurs, many people lose their pregnancies." (Mariam, mother, semi-rural Uganda)

Stillbirths were frequently associated with witchcraft; this could occur directly as result of a woman's involvement and her 'possession' by evil spirits, indirectly from 'curses' imposed by other 'witches', or failure to take herbal medicines. Having a stillbirth was often regarded as a bad omen, and some parents encountered suspicion and gossip in communities which added to stigma and isolation.

"You know this is not a common thing, when it happens everyone is questioning in their mind what would have happened and they are not comfortable talking about it... and you believe that maybe your wife is a bad omen or you have been bewitched"

(Ian, father peri urban Kenya)

In both Kenya and Uganda, stillbirth was also considered to occur as a consequence of parent's immoral behaviour. There were strong views that stillbirths were associated with infidelity or extra marital affairs:

"This man went to see his wife and from there he went to see his 'side chick' [girlfriend] and she prepared tea for him and he took it to his pregnant wife in hospital and according to customs it is not right. And when you mix that way death must occur". (Eric, father peri-urban Kenya)

Several women and partners recalled hearing similar rumours circulating in their communities after the death of the baby which caused considerable distress. Some had heard of relationship breakdown and abandonment occurring as a result. Beliefs linking stillbirth with immorality were also shared by some health workers, potentially creating negative perceptions of parents experiencing the death of a baby:

"...I understand that if you sleep with another man and you are pregnant then you will miscarry the baby or something bad will happen to the baby."

(Tamara, health worker, Kenya)

\section{Discussion}

This study has provided a uniquely in-depth account of the impact of cultural beliefs and practices on parent's experiences in the initial days and weeks after the death of their baby before or during birth in Kenya and Uganda. We have confirmed the fundamental significance of cultural norms, intertwined with spiritual and social factors in influencing understanding, behaviour and support provided to parents after their baby died. Kinship ties, particularly relationships with the mother 
and father's own parents, siblings, and in-laws (consanguineous and affinal kin) [17], provided the most powerful sources of support after the death of a baby. However, these crucial networks could be disrupted, for example by rural-to-urban migration, increasing isolation and loneliness for parents. The influence of wider communities was more mixed; norms surrounding the diminished status of stillborn babies and relative lack of rituals and mourning tended to undermine parents' grief. Parents also described pressure to conform to traditional practices and the conflicts which arose if they chose to deviate from these for example to have contact with the dead baby. These tensions also impacted health workers' practices in the period after the baby's death. Although religious fatalism provided comfort for some parents, other common explanatory factors for stillbirth including supernatural forces, notably witchcraft and immoral behaviour, reinforced stigma and taboo.

The current study demonstrated that views and norms surrounding stillbirth in Kenya and Uganda were shaped by religion and traditional beliefs, alongside knowledge from modern medicine. These influences were often combined to underpin personal and wider community beliefs and practices. Falade (2019), highlights multiple and potentially competing sources of cultural authority in African societies, where high levels of individual commitment to Christianity or Islam co-exist with adherence to traditional rituals and are equally, if not more influential than science [18]. Close family relationships provided important and consistent sources of support to many parents, the primary reliance on relatives or close friends for emotional support after stillbirth is common across other low and high-income settings [19-22]. In Kenya and Uganda, material and financial support from families was often also required. Migration and urbanisation appeared to fragment family ties, and in these circumstances, some women were able to create new relationships to fill these gaps, these often involved or were facilitated through churches. The important role of religious groups in providing emotional support for individuals in time of health crises in Africa is frequently reported elsewhere [23].

Whilst consanguineal and affinal kin relationships were largely perceived as supportive, wider community influences, perceptions and practices around stillbirth could be more problematic. Stillborn babies were assigned lesser status, rarely named, and rapidly buried often without public mourning or parents' presence. Similar practices have been reported in other LMICs settings including India [24] and had negative impacts, causing distress for parents who felt unable to express their grief openly. Kiguli et al. (2015) similarly described bereaved women in rural Eastern Uganda as 'weeping in silence' to maintain secrecy around the death of their baby [10]. Taboos around having contact with dead babies, deeply rooted in traditional beliefs in negative impacts on fertility and future successful pregnancy outcome prevented parents asking to see their baby, several later expressed unhappiness that this had not happened. Our findings demonstrate that health workers' reluctance to challenge these conventions prevented offering choices to women and families, who were denied fleeting opportunities to create memories. Although some controversy exists over benefits, studies in HIC demonstrate that in the context of compassionate care, seeing and holding stillborn babies was associated with less subsequent psychological distress and that few women who chose to see their baby regretted doing so [25].

In addition to invalidating experiences, common explanatory beliefs often reinforced stigma, which occurs when individuals are labelled and stereotyped leading to isolation, status loss and discrimination [26]. Stillbirth was often seen as evidence of poor behaviour, particularly immorality or malevolent forces. Mothers, fathers and, sometimes, the wider family experienced public stigma as a result of stillbirth and particularly after multiple baby deaths. Self-stigma was also evident, where the fear of adverse community reactions after the death of the baby led women, in particular, to develop negative feelings which could decrease self-esteem and enhance social isolation [27]. Motherhood is identified as key expectation and central role for women in African societies and perinatal death, particularly where repeated, has been a trigger for marginalisation, relationship breakdown and sporadically, domestic abuse [28]. Similar to Haws et al's (2010) study in rural Tanzania, fatalistic beliefs in these communities relieved bereaved parents of some responsibility for the death of the baby, but only if they conformed to other conventions and expectations around conduct and behaviour [29].

\section{Strengths and limitations}

Despite the importance of cultural beliefs in influencing coping and adjustment after stillbirth and huge potential psychological and social costs, few studies have addressed this issue in sub-Saharan Africa. This qualitative study explored cultural influences in a wide sample of parents, including women who birthed outside facilities and health workers in diverse urban and rural settings in Uganda and Kenya. This allowed exploration of perspectives across several tribal groups and major religious beliefs, including Christians and Muslims. However, considerable heterogeneity of norms, values and traditions affecting health behaviours have been identified within and beyond these two countries in sub-Saharan Africa [30], therefore our findings may not represent 
all East 'African' or 'African' experiences. Increasing facility-based birth means that health workers have assumed a crucial role in supporting women and families in the immediate period after stillbirth, therefore inclusion of their experiences here is a strength. Wider perspectives including those of community and religious leaders could enrich understanding further, nevertheless, feedback from National Institute for Health Research (NIHR) Stillbirth community engagement and stakeholder groups [14] in Malawi, Tanzania, Zambia and Zimbabwe demonstrated strong resonance with themes identified here, suggesting transferability to other African settings.

\section{Conclusion}

Culture has a complex and powerful influence on parents' experiences after the death of their baby in Kenya and Uganda. Aspects of African collectivist culture, such as close relationships amongst families were beneficial in enhancing support to enable parents to adjust after the trauma of stillbirth. However other norms and practices had more harmful impacts perpetuating stigma around stillbirth which is widely recognised to increase negative psychological and social outcomes for affected individuals. Stigma and fatalism also prevent open discussion of factors influencing perinatal death in communities, which has led to decades of neglect in driving improvement in stillbirth rates and care for parents in LMICs. Raising awareness of health consequences and challenging aspects of culture which have deleterious effects will be important to improve support for parents. This is a very sensitive issue, therefore interventions which promote collaboration and building trust with key stakeholders in communities are likely to be the most helpful. For example, community level education, engagement with local policy makers and religious leaders has been beneficial in challenging stigma and increasing uptake of programmes to prevent mother to child transmission of HIV in Sub Saharan Africa [31]. More sustainable progress may be achieved through strategies which accommodate positive health behaviours in the cultural framework, as opposed to direct criticism or dismissal [18].

\section{Abbreviations}

CEl: Community Engagement and Involvement; HIC: High- Income Country; LMIC: Low- and Middle-Income Country; NIHR: National Institute for Health Research; VHT: Village Health Team.

\section{Acknowledgements}

We thank all the participants for taking time to participate in this study. We acknowledge the administration at the study sites for their support during data collection. Special thanks to the CEl groups in Uganda and Kenya for reviewing the study protocol, data collection tools and assisting with confirmation of the findings.

\section{Authors' contributions}

Initial conceptualisation of the study and proposal writing TM and TL with the contribution of EA, GO and SW. EA, GO, SW, JM, AN, ANe, RM participated in data collection and initial data analysis with input from TM and RW. BSK a member of the Uganda CEI group contributed to development of study tools, data collection and analysis. All authors contributed to interpretation of the data. EA and TM drafted the manuscript with input from SW. All authors reviewed and approved the final manuscript.

\section{Funding}

This research was funded by a Wellbeing of Women/RCM/Burdett Trust International Fellowship (IFA 200) and by the National Institute for Health Research (NIHR; 16/137/53) using UK aid from the UK Government to support global health research. The views expressed in this publication are those of the author(s) and not necessarily those of the NIHR or the UK Department of Health and Social Care. The study was conducted in collaboration with Makerere University, Uganda and University of Nairobi, Kenya. The funding body had no role in the design, collection, analysis of interpretation of the data.

\section{Availability of data and materials}

The data sets used and/or analysed during the current study are not publicly archived but are available from the corresponding author on reasonable request.

\section{Declarations}

Ethics approval and consent to participate

The study was approved by the Research and Ethics Committees of; The University of Manchester UREC 2017-0233-4462; Makerere University School of Health Sciences SHSREC 2017-097: 27/04/2018 and University of Nairobi P240/05/2017: 23/06/2017. Ethical approval was also obtained from the Uganda National Council for Science and Technology SS 4666:12/07/2018. Administrative clearance was also sought from the respective hospitals. Written informed consent was obtained from all participants, including for anonymised verbatim quotes. All study processes including recruitment, data collection and data processing were carried out in accordance with relevant guidance and regulations.

\section{Consent for publication}

Not applicable.

\section{Competing interests}

The authors declare that they have no competing interests.

\section{Author details}

${ }^{1}$ Department of Nursing, College of Health Sciences, Makerere University, P.O.BOX. 7072, Kampala, Uganda. ${ }^{2}$ Centre for Childbirth, Women's and Newborn Health, Department of International Public Health, Liverpool School of Tropical Medicine, Pembroke Place, Liverpool L3 5QA, United Kingdom. ${ }^{3}$ Lugina Africa Midwives Research Network, Department of Nursing, College of Health, Makerere University, PO Box 7072, Kampala, Uganda. ${ }^{4}$ Lugina Africa Midwives Research Network, C/o School of Nursing Sciences, University of Nairobi, PO Box 30197, Nairobi, Kenya. ${ }^{5}$ Department of Geography and Environmental Science, Liverpool Hope University, Hope Park, Liverpool L16 9JD, UK. ${ }^{6}$ School of Nursing Sciences, University of Nairobi, PO Box 30197, Nairobi, Kenya. ${ }^{7}$ Mulago Specialised Women's and Neonatal Hospital, P.O. Box 7051, Kampala, Uganda.

Received: 22 March 2021 Accepted: 27 May 2021

Published online: 25 June 2021

References

1. Stillbirths. http://www.who.int/maternal_child_adolescent/epidemiolo gy/stillbirth/en/.

2. Blencowe H, Cousens S, Jassir FB, Say L, Chou D, Mathers C, Hogan D, Shiekh S, Qureshi ZU, You D, et al. National, regional, and worldwide 
estimates of stillbirth rates in 2015, with trends from 2000: a systematic analysis. Lancet Glob Health. 2016;4(2):e98-108.

3. Mills T, Ayebare E, Mukhwana R, Mweteise J, Nabisere A, Nendela A, et al. Parents' experiences of care and support after stillbirth in rural and urban maternity facilities: a qualitative study in Kenya and Uganda. BJOG. 2021;128(1):101-9.

4. Cacciatore J. Psychological effects of stillbirth. Semin Fetal Neonatal Med. 2013;18(2):76-82.

5. Berger KJ, Williams MB: Fundamentals of nursing: Collaborating for optimal health, vol. 1. Connecticut: Appleton and Lange; 1999.

6. Idang GE. African culture and values. Phronimon. 2015;16:97-111.

7. Conrad P, Barker KK. The social construction of illness: key insights and policy implications. J Health Soc Behav. 2010;51:S67-79.

8. Gire J. How death imitates life: Cultural influences on conceptions of death and dying. Online Readings Psychol Cult. 2014;6(2):3.

9. Murphy S, Cacciatore J. The psychological, social, and economic impact of stillbirth on families. Semin Fetal Neonatal Med. 2017;22(3):129-34.

10. Kiguli J, Namusoko S, Kerber K, Peterson S, Waiswa P. Weeping in silence: community experiences of stillbirths in rural eastern Uganda. Glob Health Action. 2015:8:24011.

11. Ministry of Health. Uganda Clinical guidelines: National guidelines for management of common conditions. Kampala; 2016.

12. Kiguli J, Munabi IG, Ssegujja E, Nabaliisa J, Kabonesa C, Kiguli S, Josaphat B. Stillbirths in sub-Saharan Africa: unspoken grief. Lancet (London, England). 2016;387(10018):e16-8.

13. Global Health Observatory. https://www.who.int/data/gho.

14. Bedwell C, Lavender T. Giving patients a voice: implementing patient and public involvement to strengthen research in sub-Saharan Africa. J Epidemiol Community Health. 2020;74(4):307-10.

15. Moser A, Korstjens I. Series: Practical guidance to qualitative research. Part 3: Sampling, data collection and analysis. Eur J Gen Pract. 2018;24(1):9-18,

16. Braun V, Clarke V. Using thematic analysis in psychology. Qualitaitve research in psychology. 2006:3(2):77-101.

17. Dykstra P. Kin relationships. Encyclopedia of human relationships. Volume 1, edn. Reis HT, Sprecher S (editors). Thousand Oaks: Sage; 2009: 952-954.

18. Falade B. Religious and traditional belief systems co-exist and compete with science for cultural authority in West Africa. Culture of Science. 2019;2:9-22.

19. Endo K, Yonemoto N, Yamada M. Interventions for bereaved parents following a child's death: A systematic review. Palliat Med. 2015;29(7):590-604

20. Fernandez-Sola C, Camacho-Avila M, Hernandez-Padilla JM, FernandezMedina IM, Jimenez-Lopez FR, Hernandez-Sanchez E, Conesa-Ferrer MB,
Granero-Molina J. Impact of Perinatal Death on the Social and Family Context of the Parents. Int J Environ Res Public Health. 2020;17(10):3421.

21. Heazell AE, Siassakos D, Blencowe H, Burden C, Bhutta ZA, Cacciatore J, Dang N, Das J, Flenady V, Gold KJ, et al. Stillbirths: economic and psychosocial consequences. Lancet. 2016;387(10018):604-16.

22. MCNeil MJ, Namisango E, Hunt J, Powell RA, Baker JN. Grief and Bereavement in Parents After the Death of a Child in Low- and Middle-Income Countries. Children (Basel). 2020;7(5):39.

23. Manderson L, Block E. Relatedness and care in Southern Africa and beyond. Soc Dyn. 2016;42:205-17.

24. Roberts LR, Montgomery S, Lee JW, Anderson BA. Social and cultural factors associated with perinatal grief in Chhattisgarh India. J Community Health. 2012;37(3):572-82.

25. Cacciatore J. The silent birth: a feminist perspective. Soc Work. 2009:54(1):91-5.

26. Link BG, Phelan JC. Conceptualizing Stigma. Ann Rev Sociol. 2001;27:363-85.

27. Pollock D, Ziaian T, Pearson E, Cooper M, Warland J. Understanding stillbirth stigma: A scoping literature review. Women Birth. 2020:33(3):207-18.

28. Shakespeare C, Merriel A, Bakhbakhi D, Baneszova R, Barnard K, Lynch M, Storey C, Blencowe H, Boyle F, Flenady V, et al. Parents' and healthcare professionals' experiences of care after stillbirth in low- and middleincome countries: a systematic review and meta-summary. BJOG. 2019;126(1):12-21.

29. Haws RA, Mashasi I, Mrisho M, Schellenberg JA, Darmstadt GL, Winch PJ. "These are not good things for other people to know": how rural Tanzanian women's experiences of pregnancy loss and early neonatal death may impact survey data quality. Soc Sci Med. 2010;71(10):1764-72.

30. Ogundipe R. Cultural practices and health consequences' in and Awofeso, $\mathrm{N}$ (eds) Public Health in developing countries London: Intech Open. In: Public Health in developing countries edn. Agnugwom E, Awofeso N (Editors). London: Intech Open; 2020.

31. Sripipatana T, Spensley A, Miller A, McIntyre J, Sangiwa G, Sawe F, Jones D, Wilfert CM. Site-specific interventions to improve prevention of motherto-child transmission of human immunodeficiency virus programs in less developed settings. Am J Obstet Gynecol. 2007;197(3 Suppl):S107-112.

\section{Publisher's Note}

Springer Nature remains neutral with regard to jurisdictional claims in published maps and institutional affiliations.
Ready to submit your research? Choose BMC and benefit from:

- fast, convenient online submission

- thorough peer review by experienced researchers in your field

- rapid publication on acceptance

- support for research data, including large and complex data types

- gold Open Access which fosters wider collaboration and increased citations

- maximum visibility for your research: over 100M website views per year

At BMC, research is always in progress.

Learn more biomedcentral.com/submissions 